\title{
Quality Of Life and Satisfaction with Care among Breast Cancer Survivors Receiving Different Treatments Strategies in Sri Lanka
}

\author{
Munasinghe $\mathrm{WH}^{1 *}$, Nanayakkara $\mathrm{PGCL}^{2}$ and Rathnayaka $\mathrm{N}^{1}$ \\ ${ }^{1}$ Allied Health Science Degree Programme, University of Ruhuna, Srilanka \\ ${ }^{2}$ Department of Anatomy, University of Ruhuna, Srilanka
}

Submission: September 24, 2016; Published: October 03, 2016

"Corresponding author: WasanaHarshani Munasinghe, BSC nursing degree, Undergraduate, Faculty of medicine, University of Ruhuna, Sri Lanka, Tel: 071-9021909; Email: wasanahmunasinghe@gmail.com

\begin{abstract}
Introduction and Objective: This study was aimed to evaluate the QOL among BCS who receiving various treatment strategies in Teaching Hospital, Karapitiya (THK).

Method: This cross sectional study was performed with conveniently selected 142 BCS (Mean age (SD) - 55 (10.29) years) at oncology unit of THK during July 2016 using WHOQOL-BREFF questionnaire.

Results: Among the studied BCS, 32.4\% were studied up to 0/L and $61.3 \%$ were married. $76.8 \%$ were unemployed and $38.7 \%$ had monthly income between Rs 10,000-30,000. 51.4\% were living with their husband and children and 93\% were Buddhist. 34.5\% had undergone to CT. Mean (SD) of physical (PD), psychological (PsD), social (SD) and environmental (ED) domains were 47.79 (11.20), 42.17 (15.19), 48.86 (15.68) and 51.83 (13.61) respectively. Age, marital status, level of education and monthly income and current treatments were significantly affected to their QOL. But CFs had no significant impact on QOL. Generalized body pain, insomnia, vomiting, fatigue, tingling sensation or numbness of legs or hands, problem of testy with food were significantly affected to their QOL. Majority of BCS satisfied with their family support and support provided by HCW. Monthly income, level of education, marital statuses were significant with their satisfaction on family support and monthly income was significant with their satisfaction on the support provided by the health care HCW. Satisfaction with health care and family support had significant impact on QOL of BCS.
\end{abstract}

Conclusion: Physical and psychological domains were impaired mostly among BCS. SDF, current treatment strategies, side effect of treatments, satisfaction with health care provided and family care significantly affected BCS's QOL. SDF had impact on BCS's satisfaction with health care provided and family care. Modifiable factors such as improvement of side effects and immense care for their satisfaction will be enabling to empower the QOL of BCS.

Keywords: Quality of Life; Breast cancer survivors; Socio-demographic factors; Clinical factors; Health Care Workers

Abbreviations: BCS: Breast Cancer Survivors; WHO: World Health Organization; QOL: Quality of Life; HCW: Health Care Workers; THK: Teaching Hospital Karapitiya

\section{Introduction}

Now a day, cancers have become a critical health issue worldwide which create numerous problems to the health domains of individuals. Cancer Research UK [1]. Among these cancers, breast cancer is the most common cancer in women Cdc.gov [2]. According to most recent statistics available in Sri Lanka, the incidence of breast cancer among female population is 18.8 per 100,000 populations and 60-64 years old female population is the most vulnerable age group for breast cancer (cancer incidence data Sri Lankan year [3] During the last decade, survival rates for breast cancer have increased as a result of earlier detection and increased use of adjuvant therapy
Macmillan.org.uk [4]. Women receive radiation therapy and/or chemotherapy along with systemic hormonal therapy for breast cancer treatment depending on the stage and estrogen receptor status at diagnosis.

Diagnosis of disease and starting treatments, its side effects are directly affected to their QOL. Therefore, it is important for health care professionals to become familiar with QOL of Breast Cancer Survivors (BCS). QOL is a broad, multidimensional concept that lacks a precise definition in the medical literature. According to the World Health Organization (WHO), quality of life is individuals" perception of their position in life in the context of the culture and value systems in which they live and 


\section{Cancer Therapy \& Oncology International Journal}

in relation to their goals, expectations, standards, and concerns" (WHO, 1993). With the diminished QOL among the cancer patients, their level of satisfaction has a greater effect. The way health care workers (HCW) caring them and family members caring to them directly affected to the prognosis and also to spend their remaining life in a satisfactory manner, if they are suffering in critical type of cancer. The aim of this study was to assess QOL and satisfaction with health care and family care among BCS receiving different treatments strategies.

\section{Material and Methods}

This study was a cross-sectional study of 142 convenient samples of BCS that were admitted for any type of treatment in the oncology unit of Teaching Hospital Karapitiya between May to August 2016. It is a major oncology unit in the southern province of Sri Lanka. Any patients with breast cancer, age between 18 yrs to $80 \mathrm{yrs}$, under any type of treatment were eligible to enter the study.

The exclusion criteria were; patients had chronic diseases, hearing and speaking disabilities previous and concurrent malignancies.

Interviewer administered, semi structured questionnaire with both closed ended and open ended questions were used to collect the data on the current topic. The questionnaire includes seven sections as, socio-demographic information, status of cancer, treatments modality evaluation, assessments of side effect of treatments, assessments of QOL and satisfaction with care.

Socio-demographic data included age, marital status, level of education, monthly income, occupational and living status and clinical data including age of diagnosis, duration of disease, stage of carcinoma, type of treatments, its side effects.

WHO-BREEF Sinhala version was used to assess the QOL of BCS by Somasiri KG, Gunawardhana S, et al. [5] QOL measurement instrument is a short version of a QOL assessment instrument WHOQOL-100 (WHO, 1997). WHOQOL-BREF which provides a fast profile of 4 areas (domains). From those 26 questions, 2 questions were related to general health and overall QOL; and the following 24 questions provided a broad and comprehensive assessment of the QOL of a patient each question of the WHOQoLBREFF instrument had five of the answer choice on an ordinal likert scale. All of them produced a profile of four domains; physical, psychological, social relationship and the environment (WHO, 1997). Investigator developed questions were used to assess BCS's satisfaction with health and family care [6].

The study was approved by the ethical committee of the Faculty of Medicine, University of Ruhuna. Before interview survey, the interviewer explained the purpose of these questions to all eligible individuals and requested their participation.

Data were presented by percentages and tables (Tables 1-4). One way -ANOVA test was used to assess the difference between categorical variables. Statistical significance was set at $\mathrm{p}<0.05$. All calculation performed using SPSS 20 version.

Table 1: Difference between physical, psychological, social and environmental domains with socio-demographic variables and ongoing treatments.

\begin{tabular}{|c|c|c|c|c|}
\hline \multirow{2}{*}{ Variables } & \multicolumn{4}{|c|}{ WHO-BREFF } \\
\cline { 2 - 5 } & Physical domain & Psychological domains & Social domain & Environmental domain \\
\hline Age & 0.28 & $0.03^{*}$ & 0.98 & 0.59 \\
\hline Marital status & 0.43 & 0.12 & 0.63 & $0.002^{* *}$ \\
\hline Level of education & 0.09 & 0.22 & 0.33 & $0.001^{* *}$ \\
\hline Monthly income & 0.12 & 0.18 & $0.04^{*}$ & $0.001^{* *}$ \\
\hline Occupational status & 0.65 & 0.27 & 0.12 & 0.71 \\
\hline Living status & 0.45 & 0.38 & 0.69 & 0.16 \\
\hline Ongoing treatments & $0.002^{* *}$ & 0.71 & 0.13 & 0.68 \\
\hline
\end{tabular}

$\mathrm{p}<0.05^{\star} ; \mathrm{p}<0.01^{* *}$

Table 2: Satisfaction categories based on the family support score and health care services score.

\begin{tabular}{|c|c|c|c|}
\hline Care & Dissatisfied & Satisfied & Highly satisfied \\
\hline Family support & $<35$ & $36-40$ & $41<$ \\
\hline HCW support & $<30$ & $31-34$ & $35<$ \\
\hline
\end{tabular}

Table 3: Difference of socio-demographic status on satisfaction with family support and support provided by HCW.

\begin{tabular}{|l|c|c|c|c|c|c|c|}
\hline & Age & Marital status & Level of education & Monthly income & $\begin{array}{c}\text { Occupational } \\
\text { status }\end{array}$ & Religion & Living status \\
\hline Family care & 0.18 & $0.02^{*}$ & $0.001^{* *}$ & $0.008^{* *}$ & 0.59 & 0.35 & 0.08 \\
\hline Health care & 0.99 & 0.61 & 0.22 & $0.001^{* *}$ & 0.95 & 0.93 & 0.30 \\
\hline
\end{tabular}

$P<0.05^{*} ; p<0.01^{* *}$ 


\section{Cancer Therapy \& Oncology International Journal}

Table 4: Influence of satisfaction of family and health care services on QOL.

\begin{tabular}{|c|c|c|c|c|}
\hline & Physical & Psychological & Social & Environmental \\
\hline $\begin{array}{c}\text { Family } \\
\text { support }\end{array}$ & $0.04^{*}$ & $0.002^{* *}$ & $0.02^{*}$ & $0.002^{* *}$ \\
\hline $\begin{array}{c}\text { Health } \\
\text { support }\end{array}$ & $0.001^{* *}$ & 0.08 & 0.25 & $0.01^{*}$ \\
\hline
\end{tabular}

\section{Results}

The study sample compromised 142 BCS who were receiving different treatment strategies in THK. The mean (SD) age of the BCS was 55 (10.29). 32.4\% (46) of them were studied up to 0/L and 87 (61.3\%) of them were married. 103 (76.8\%) participant were unemployed (housewives) and 55 (38.7\%) participants had a monthly income ranging from Rs 10,000-30,000. 51.4\% of them are living with their husband and children 93\% were Buddhist. Majority of the study participants diagnosed their condition when they 5 th decade of their lives $(45,31.7 \%)$ and $37.3 \%$ (53) were in the $2^{\text {nd }} 6$ months of their disease duration. $54.2 \%$ had spread to adjacent lymph nodes and $26.1 \%$ had distance metastasis of their breast cancer. During study period, $34.5 \%$ women were receiving chemotherapy. The most severely affected side effects after different treatment modalities were generalized body pain (40.8\%), anorexia (58.5\%), fatigue (38.7\%), hot flushes and/or sweat (32.4\%), problem with food's tasty or spicy food (52.1\%) and hair loss (55.6\%).

Mean (SD) of physical domain was 47.79 (11.20), psychological domain was 42.17 (15.19), social domain was 48.86 (15.68) and environmental score 51.83 (13.61) respectively. Environmental and social domains had higher mean score than physical and psychological domains.

Physical domain had no significant differences among the different socio-demographic characteristics $(p>0.05)$. However, psychological domain was significantly differ among different age categories $(\mathrm{p}=0.03)$ and environmental domain was significantly differ among different groups of marital status $(\mathrm{p}=0.002)$, level of education $(p=0.001)$ and monthly income $(p=0.001)$ and social domain was significantly differ among different groups of monthly income $(p=0.04)$. Other hand, there was no significant difference between QOL domains and status of cancer. Physical domain was significantly different among the ongoing treatment strategies $(p=0.002)$ and there were no significant differences between current treatment options and other QOL domains among the study participants ( $\mathrm{p}>0.05)$.

Generalized body pain ( $p=0.018)$, insomnia $(p=0.005)$, vomiting $(\mathrm{p}=0.005)$, fatigue $(\mathrm{p}=0.008)$, tingling sensation or numbness of legs or hands $(\mathrm{p}=0.024)$ and problem with tasty of food $(p=0.006)$ were significantly different with physical domain. Anorexia $(p=0.019)$, tingling sensation or numbness of legs or hand $(p=0.001)$, problem with testy of food $(p=0.036)$ were significantly different with psychological domain. Problem with tasty with food and spicy food $(\mathrm{p}=0.013)$ and anorexia $(p=0.01)$ had statically significant difference with social domain. Environmental domain were significantly different with tingling or numbness sensation of hand and/or foot $(\mathrm{p}=0.03)$.

Participants were categorized in to three groups using their scores for satisfactions with family support and health care provided.

Generally, most of the participants were satisfied about health care services and their family support. 68 (47.9\%) participant were satisfied with their family support and 52 $(36.6 \%)$ patients were satisfied with support given by the health care workers.

Monthly income $(p=0.008)$, level of education $(p=0.001)$ and marital status $(\mathrm{p}=0.02)$ had significant differences with family support. Monthly income ( $\mathrm{p}=0.001)$ had significant difference with satisfaction with the support provided by the HCW.

There was significant association between satisfaction with family care and physical, psychological, social and environmental domains. When considering health care services, there was significant association between satisfaction with health services and physical and environmental domain.

\section{Discussion}

Quality of life (QOL) assessments have found acceptability among physicians, nurses, and psychosocial staff for the several reasons. Assessments of QOL benefit for Breast Cancer Survivors (BCS) as it provide some insight into their life. They provide insights into life domains affected by breast cancer that are usually unaddressed, including a patient's mental health, emotional well-being, family and social relations, and abilities to maintain a career, uphold finances, and pursue leisure activities. (Perry, Kowalski and Chang, 2007) According to the current study results, the four QOL domains analyzed on this study, environmental domain showed the highest score and the psychological domain had the lowest score. Barrios et al. [7] reported poor QOL in BCS in panama in all four domains Barrios [7]. Similar findings were observed in a study done in Jordan as well where they showed poor QOL among patients who are receiving adjuvant treatments Alzabaidey [8]. When considering the social and physical domain, physical score showed a lower score than the social domain. In this study most of the participants were physically and psychologically get affected in their quality of life. QOL on social and environmental domains were high in the current study. The reasons behind this finding may be the cultural background, where females are more dependent on the others in the family, females' worrying more about the family and other issues in the family. Also, different socio-economic backgrounds may affect in these variations.

According to the finding of this study, age was significantly affected to the psychological score domain $(p=0.03)$ in women. Where younger BCS had badly affected psychologically due to their disease. Similar finding were reported by a study done by 


\section{Cancer Therapy \& Oncology International Journal}

Anderson et al, with younger BCS (<50years) had worse outcome of depressive symptoms. They found the reasons for this result were as pre mature menopause, menopausal symptoms, and infertility. The latter was common in younger women and had a role in the level of distress after treatments.

In our settings, Income was a major factor affecting the BCS. As Sri Lanka is a developing country, most of the participants had poor income level. Brest cancer is a life threating illness and after diagnosis and starting of treatments it would directly affect to their income. Most of the participants came from rural areas. Therefore, they have to spend a lot of money for their transport facilities. In addition to that, all medications and investigations were not available in our setting. Therefore, they have to spend a lot of money for investigation and medication. As this study revealed, lower income level had directly affected to their QOL.

Among four domains of quality of life, social $(p=0.04)$ and environmental $(\mathrm{p}=0.001)$ domains showed statistically significant with monthly income. Earlier studies also revealed that monthly income was statistically significant with QOL domains Safaee, Tatar \& Somunoğlu [9], zou et al. [10]. In this current study, marital status ( $p=0.002)$ and level of education $(p=0.001)$ showed a statistically significant with the environmental score. This study finding is supported by another study where the marital and educational statuses were significantly affected to their QOL (Awadalla et al. 2007). According to this, occupational $(\mathrm{P}>0.05)$ and living statuses $(\mathrm{p}<0.05)$ were not affected to their quality of life. Having a partner did not produce significant difference to their QOL. This finding was supported by a study done in Panama; where no significant deference was found between QOL domains and status of living of women Barrios [7].

When comparing the score of quality of life domain and clinical variables (age of onset the illness, stage of cancer, and duration of the illness) no statistically significant difference was found. A study done in Iran has shown the duration of disease and grade of tumor had a significant difference with the QOL score. Those with duration of disease less than four months reported significantly lesser global quality of life scores Safaee et al. [9]. But instrument which was used for the data collection was QLQ-C30. Similar study done by Awadalla et al. [11] showed the duration of illness was statistically significant with the QOL.

Among side effects, more severely affected side effects after treatments were the generalized body pain (40.8\%), insomnia (26.1\%), anorexia (58.5\%), fatigue $(38.7 \%)$, and pain in lower back $(16.9 \%)$, tingling or numbness in hands or feet $(40.1 \%)$, hot flushes and/or sweats (32.4\%), problems with food's tasty or spicy food (52.1\%), hair lose (55.6\%). Fatigue was the commonest side effect among BCS of this study. According to the previous study, more common problems were the anorexia and fatigue in patients receiving chemotherapy Alzabaidey [7]. Similarly in our study, most of the participant had a currently receiving chemotherapy and complained fatigue and anorexia. Results indicate, anorexia ( $p=0.02)$, tingling or numbness of legs or hand ( $p=0.001)$ and problem with tasty of food $(p=0.03)$ had a statistically significant difference with the psychological domain and generalized body pain $(p=0.02)$, insomnia $(p=0.005)$, vomiting $(p=0.005)$, fatigue $(p=0.008)$, tingling or numbness of legs or hands $(p=0.001)$, problem with testy of foods $(p=0.006)$ had significant difference with physical score domains. Even although the most of the participants complained about hair loss during their chemotherapy, there was no statistically significant difference with QOL domains. Anorexia $(\mathrm{p}=0.01)$ and problem with tasty of food $(\mathrm{p}=0.013)$ had a significant difference with social score domain and tingling or numbness of hands or legs $(p=0.03)$ had a significant with environmental score domain.

In this study, ongoing treatments $(\mathrm{p}=0.002)$ were statistically significant with their physical score domain. This study finding is similar to a study by, (Kyei et al. 2014), where patients who had radiotherapy had a highest QOL score than patients who had chemotherapy. A study done by Gokgoz et al. [12], they showed that the patients who were currently receiving chemotherapy had a lower global health QOL than from those who receiving hormone therapy. Furthermore, a study done in Jordan, assessing QOL of BCS receiving adjuvant therapy reported that a significant difference found between physical score $(p=0.001)$ and treatments and but there was no significant difference psychological score $(\mathrm{p}=0.621)$ and treatments strategies Alzabaidey [5].

During this study, we assessed patients' level of satisfaction with the family care and the health care provided. Participants divided to three groups using their satisfaction score. Most of the participants were satisfied with their family support (47.9\%) and health care provided (36.6\%). Otherwise, 52(36.6\%) were very dissatisfied with their family support and $46(32.4 \%)$ were very dissatisfied with their health care services provided. Regarding family support for the BCS, it was affected to their four domains of quality of life. But health care provided affected only on their physical and social domain. Regarding sociodemographic data, marital status affected on satisfaction with health care provided and marital status, level of education and monthly income affected on the satisfaction with family support. A study done in Sri Lanka by Mudduwa and Punchihewa [13] showed a high proportion of patients receiving satisfactory family support to cope with the illness. Similarly, study done in panama, satisfaction with family support and health care provided had a statistically significant difference with four domain of quality of life Barrios [7]. Also a study done in Taiwan, reported that the, patients had the most dissatisfaction with the health factors HL et al. [14]. It could be due to the difference between family backgrounds, bond with other family members and the caring behavior of family members in Sri Lanka [15-17].

\section{Conclusion}

This study, mostly affected QOL domains were the physical and psychological domains among BCS. Age was associated with psychological domain. Income of the participant was associated 


\section{Cancer Therapy \& Oncology International Journal}

with the environmental and social domain. Marital status and level of education was affected on environmental domain. None of the CF was associated with QOL domains. Fatigue, generalized body pain, insomnia, vomiting, tingling or numbness sensation of legs or/and hands, problem with tasty of food were affected mostly on QOL mainly on physical domain. Anorexia, tingling or numbness of legs or/and hands and problem with tasty of food were affected on psychological domain. Anorexia and problem with testy of food were affected on social domain and tingling and numbness sensation of legs or/and hands affected environmental domain. Current treatments were affected on physical domain. Most of the participant satisfied with their family support and support provided by the HCW. Satisfaction with family support had significant impact on the four QOL domains and satisfaction with HCW was impacted on both physical and social domains of QOL.

\section{References}

1. Cancer Research UK (2014) what is cancer?.

2. Cdc.gov (2015) CDC - Breast Cancer Statistics.

3. PS G, MR S (2007) Quality of life and adjuvant systemic therapy for early-stage breast cancer. Expert Rev Anticancer Ther 7(8): 11231134.

4. Macmillan.org.uk (2015) How cancer affects people - Information and support - Macmillan Cancer Support.

5. Somasiri KG, Gunawardhana S (2003) Assessment of Quality of Life using Sinhala translation of WHO-QOL100.

6. Oliver M (1997) WHOQOL-BREEF.
7. Barrios, Mayela "Quality Of Life In Female Breast Cancer Survivor In Panama". sholar commons 1651 (2016): 18-40.

8. Alzabaidey F (2012) Quality of Life Assessment for Patients with Breast Cancer Receiving Adjuvant Therapy. Journal of Cancer Science \& Therapy 4(3).

9. Tatar M and Somunoğlu S (2012) A study on the identification of factors affecting the life quality of breast cancer patients. International Journal of Pharmaceutical and Healthcare Marketing. 6(4): 376-396.

10. Zou Z, Hu j and Mc Coy T (2014) Quality of life among women with breast cancer living in wuhan, china. International journal of nursing sciences pp.79-88.

11. Awadalla A, Ohaeri J, Gholoum A, Khalid A, Hamad H, et al. (2007) Factors associated with quality of life of outpatients with breast cancer and gynecologic cancers and their family caregivers: a controlled study. BMC Cancer 7(1): 102

12. Gokgoz S, Sadikoglu G, Paksoy E, Guneytepe U, Ozcakir A, et al. (2011) Global Journal of Health Science. Health Related Quality of Life among Breast Cancer Patients: a Study from Turkey, 3(2): 140.

13. Mudduwa l and Punchihewa G (2011) Psychological impact of breast cancer; a study done in a Sri Lankan setting. Gall medical Journal 16(1): 16-21.

14. HL l, NP K, Wj D and L P (2001) Factor related to Quality of Life in breast cancer patients receiving chemotherapy. Pubmed 9(3): 57-68.

15. Ncisl.health.govlk (2010) National Cancer Hospital - Maharagama.

16. Kayl A and Meyers C (2006) Us National Library of Medicine. Sideeffects of chemotherapy and quality of life in ovarian and breast cancer patients 18(1): 8 .

17. Safaee A, Moghimi-Dehkordi B, Zeighami B, Tabatabaee $\mathrm{H}$ pourhoeseingholi M (2008) Predictors of quality of life in breast cancer patients under chemotherapy 45(3): 107-111. 